\title{
Persistance des conidiospores des hyphomycètes entomopathogènes Beauveria bassiana (Bals.) Vuill., Metarhizium anisopliae (Metsch.) Sor., Nomuraea rileyi (F.) Samson et Paecilomyces fumoso-roseus Wize dans le sol, en conditions contrôlées
}

\author{
Jacques FARGUES \& Pierre-Henri ROBERT \\ avec la collaboration technique de Hervé Vermerl dE CONCHARD \\ I.N.R.A., Station de recherches de Lutte biologique, La Minière, F 78280 Guyancourt
}

\begin{abstract}
L'étude comparative de la persistance des conidiospores de 4 hyphomycètes pathogènes d'insectes a été menée en conditions contrôlées dans un sol expérimental à $19^{\circ} \mathrm{C}$. Les inoculums des souches Metarhizium anisopliae $\mathrm{N}^{\circ} 32$, Nomuraea rileyi $\mathrm{N}^{\circ} 5$, Paecilomyces fumoso-roseus $\mathrm{N}^{\circ} 39$ et surtout Beauveria bassiana $\mathrm{N}^{\circ} 18$ se dégradent en moins d'un an (pertes pondérales de matière sèche de 70-80 p. 100 en 4 à 6 mois, potentiel d'inoculum négligeable). En revanche, la stabilité des conidies de l'isolat $M$. anisopliae $\mathrm{N}^{\circ} 51$ est remarquable (perte de biomasse de 5,4 p. 100 en 21 mois). L'analyse du potentiel infectieux des inoculums des 2 souches de $M$. anisopliae confirme leur différence d'aptitude à la survie dans le sol. La persistance des conidiospores est donc une propriété inhérente à chaque souche ; dans le cas de l'isolat $M$. anisopliae $\mathrm{N}^{\circ} 51$, elle pourrait résulter
\end{abstract} d'une évolution de l'inoculum en cours d'incubation.

Mots clés additionnels : Biomasse, potentiel d'inoculum, potentiel infectieux, technique de pièges. Vuill., Metarhizium anisopliae (Metsch) Sor., Nomuraea rileyi (F.) Samson and Paecilomyces fumoso-roseus Wize, in controlled conditions.

The persistence of conidia of 4 entomopathogenic hyphomycetes in soil was investigated with an experimental biodegradation method using a trap technique at $19^{\circ} \mathrm{C}$. At various times of incubation, traps were collected to study changes in biomass and inoculum potential. The infection potential of 2 Metarhizium anisopliae isolates was also tested on scarabeid larvae. Inocula of $M$. anisopliae $\mathrm{N}^{\circ} 32$, Nomuraea rileyi $\mathrm{N}^{\circ} 5$, Paecilomyces fumoso-roseus $\mathrm{N}^{\circ} 39$ and Beauveria bassiana $\mathrm{N}^{\circ} 18$ were substantially degraded after 6 months incubation (70$80 \%$ dry weight loss). In contrast, the activity of $M$. anisopliae $\mathrm{N}^{\circ} 51$ remained at the initial level after 21 months. The analysis of the respective infection potential of biodegraded conidia of both strains of $M$. anisopliae confirmed their difference of stability. Thus, all investigations demonstrated that the persistence of conidia depends on the fungal strain. In addition, microcyclic conidiation could be implicated in the high survival of conidia of $M$. anisopliae $\mathrm{N}^{\circ} 51$.

Additional key words : Biomass, inoculum potential, infection potential, trap technique.

\section{INTRODUCTION}

La plupart des auteurs s'accordent pour attribuer au sol un rôle de réservoir des champignons entomopathogènes, qu'il s'agisse de mycoses d'insectes à vie larvaire souterraine (FERRON, 1978) ou épigée (IGNOFFO et al., 1978). Sur le plan épidémiologique, la persistance des propagules fongiques dans le sol présente donc un intérêt majeur (JOUSSIER, 1977; FERRON, 1978). 
Il est admis en général que la survie des conidiospores produites par ces germes peut dépasser un an en milieu édaphique (ROBERTS \& CAMPBELL, 1977). Néanmoins, certains auteurs ont noté des différences d'aptitude à la persistance des conidiospores dans le sol entre les espèces cryptogamiques pathogènes d'insectes. MÜLLER-KÖGLER \& STEIN $(1970,1976)$ montrent que le nombre de spores viables de Beauveria bassiana (Bals.) Vuill., en sol de serre, diminue de plus de 10 fois en moins d'un an alors que celui des conidies de Metarhizium anisopliae (Metsch.) Sor. baisse seulement de 30 à 50 p. 100 en 2 ans. FERRON (1971) constate que les conidiospores de Beauveria brongniartii (= tenella) (Sacc.) Petch. conservent leur potentiel infectieux pendant au moins un an à $20^{\circ} \mathrm{C}$ dans la terre, tandis que, dans la tourbe, leur virulence baisse après 6 mois, toutes conditions étant égales par ailleurs. IGNOFFO et al. (1978) rapportent que l'activité pathogène des conidies de Nomuraea rileyi (F.) Samson diminue de moitié après $100 \mathrm{j}$ d'enfouissement dans le sol.

Ces données bibliographiques diffèrent par les critères retenus (potentiel d'inoculum ou potentiel infectieux) et par les paramètres expérimentaux (nature physico-chimique du sol, conditions thermiques et hydriques, ...). C'est pourquoi nous avons entrepris l'étude comparative de la persistance des conidiospores de 4 espèces entomopathogènes, $B$. bassiana, $M$. anisopliae, $N$. rileyi et Paecilomyces fumosoroseus Wize en conditions contrôlées dans un sol expérimental. Une seule souche par espèce cryptogamique a été éprouvée, sauf dans le cas de $M$. anisopliae pour laquelle 2 pathotypes ont été retenus.

\section{MATÉRIEL ET MÉTHODE}

\section{A. Inoculums cryptogamiques}

L'expérimentation a porté sur 5 isolats conservés à la mycothèque I.N.R.A. de la Station de Recherches de Lutte biologique de La Minière :

- Beauveria bassiana $\mathrm{N}^{\circ} 18$, utilisé dans les essais de lutte biologique contre le doryphore (FARGUES et al., 1980) ;

- Metarhizium anisopliae $\mathrm{N}^{\circ} 32$ et $\mathrm{N}^{\circ} 51$, dont l'adaptation parasitaire aux larves de Scarabéides a fait l'objet d'études approfondies (FARGUES \& ROBERT, 1983) ;

- Nomuraea rileyi $\mathrm{N}^{\circ} 5$ et Paecilomyces fumosoroseus $\mathrm{N}^{\circ} 39$, retenus pour leur activité pathogène à l'égard des Noctuides (FARGUES \& RoDRIGUEZRUEDA, 1980).

Les cultures ont été réalisées en fjoles de Roux sur milieu semi-synthétique gélosé comprenant : $\mathrm{KH}_{2} \mathrm{PO}_{4}$ : $0,39 \mathrm{~g} ; \mathrm{Na}_{2} \mathrm{HPO}_{4}, 12 \mathrm{H}_{2} \mathrm{O}: 1,42 \mathrm{~g} ; \mathrm{MgSO}_{4}, 7 \mathrm{H}_{2} \mathrm{O}$ : $0,60 \mathrm{~g} ; \mathrm{KCl}: 1,00 \mathrm{~g}$; glucose : $10 \mathrm{~g} ; \mathrm{NH}_{4} \mathrm{NO}_{3}: 0,70 \mathrm{~g}$; agar : $20 \mathrm{~g}$; supplémenté en extrait de levure : $5 \mathrm{~g}$, dans $1000 \mathrm{ml}$ d'eau distillée ; $\mathrm{pH} \mathrm{6,5}$. Après 3 semaines d'incubation à $24 \pm 1{ }^{\circ} \mathrm{C}$ sous une photophase quotidienne de $16 \mathrm{~h}$, les conidiospores sont recueillies à sec par raclage superficiel du milieu de culture.

\section{B. Méthode d'étude de la persistance des germes dans le sol}

\section{Principe}

L'introduction d'un substrat inerte (minéral ou organique) ou d'un organisme vivant (végétal ou animal) dans le sol se traduit par des interactions avec les divers éléments de la microflore tellurique (DOMMERGUES \& MANGENOT, 1970 ; KILBERTUS et al., 1975). Dans le cas des propagules fongiques entomopathogènes, on a pu mettre en évidence l'apparition de processus antagonistes tels que des mycolyses d'origine bactérienne et du prédatisme par des amibes mycophages ou par certains microarthropodes (REISINGER et al., 1977 ; FARGUES et al., 1983). Nous avons donc utilisé une technique de piégeage (PARKINSON et al., 1971 ; MANGENOT \& REISINGER, 1977) consistant à placer un échantillon de spores dans un piège qui est enfoui dans un sol expérimental, l'évolution de la biomasse, du potentiel d'inoculum et du potentiel infectieux de ces propagules étant suivie au cours du temps.

\section{Description du dispositif et mode opératoire}

Les échantillons ( $6 \mathrm{~g}$ de sporée fraîche), enveloppés dans un sachet de toile à bluter à mailles fines $(30 \mu \mathrm{m})$, sont placés dans des pièges constitués d'un cylindre creux en matière plastique imputrescible $(\varnothing=6 \mathrm{~cm}$ et $\mathbf{H}=1,5 \mathrm{~cm}$ ) fermé aux extrémités par 2 disques de toile à bluter rigide (maille $500 \mu \mathrm{m}$ ) maintenus par 2 rondelles de matière plastique fixées à l'aide de 4 vis en acier inoxydable. Chaque piège est enfoui dans un cylindre garni de terre non stérile, qui est lui-même placé dans un récipient clos maintenu à $19 \pm 1{ }^{\circ} \mathrm{C}$ (fig. 1). Nous avons pris comme terre de référence un sol limoneux d'une capacité de rétention de 26 p. 100 (tabl. 1).

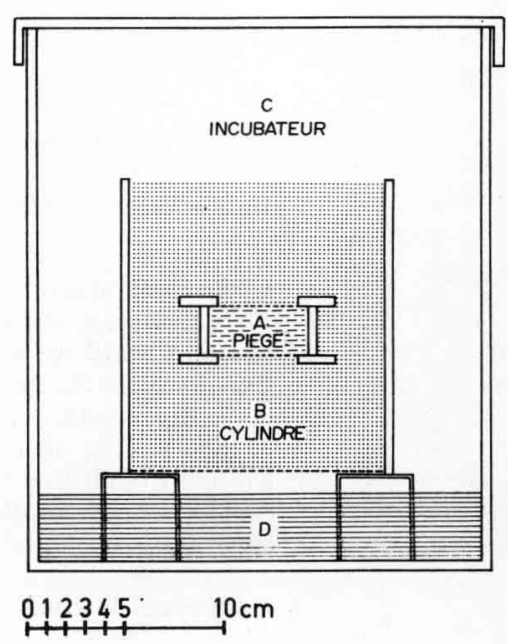

Figure 1

Dispositif expérimental pour l'étude de la persistance des conidiospores d'hyphomycètes entomopathogènes dans le sol en conditions contrôlées $\left(19^{\circ} \mathrm{C}\right.$, humidité du sol ajustée à $80 \mathrm{p} .100$ de sa capacité de rétention). Echantillons de conidies dans le piège $(A)$. Sol expérimental dans le cylindre d'incubation (B). Incubateur (C). Eau (D). Incubation unit used for studying the persistence of entomopathogenic conidia in soil under controlled conditions $\left(19^{\circ} \mathrm{C}\right.$, soil adjusted to $80 \%$ moisture-holding capacity). Sample of naked conidia in trap (A). Experimental soil (B). Incubator (C). Water (D). 


\section{TABLEAU 1}

Analyse du sol limoneux, d'une capacité de rétention de $26 \mathrm{p} .100$, utilisé dans les essais de persistance des conidiospores en milieu édaphique.

Analysis of the experimental soil (sandy loam) of $26 \%$ water retention capacity used in experiments of conidial persistence.

\begin{tabular}{|c|c|}
\hline $\begin{array}{c}\text { Analyse mécanique } \\
\text { Cailloux } \\
\text { Graviers } \\
\text { Terre fine }\end{array}$ & $\begin{array}{r}0 \\
80 \\
920\end{array}$ \\
\hline $\begin{array}{c}\text { Granulométrie sans décalcification } 8 \text { fractions p. } 1000 \\
\text { Particules comprises entre } 0,000 \text { et } 0,002 \mathrm{~mm} \\
\text { Particules comprises entre } 0,002 \text { et } 0,020 \mathrm{~mm} \\
\text { Particules comprises entre } 0,020 \text { et } 0,050 \mathrm{~mm} \\
\text { Particules comprises entre } 0,050 \text { et } 0,100 \mathrm{~mm} \\
\text { Particules comprises entre } 0,100 \text { et } 0,200 \mathrm{~mm} \\
\text { Particules comprises entre } 0,200 \text { et } 0,500 \mathrm{~mm} \\
\text { Particules comprises entre } 0,500 \text { et } 1 \mathrm{~mm} \\
\text { Particules comprises entre } 1 \mathrm{~mm} \text { et } 2 \mathrm{~mm}\end{array}$ & $\begin{array}{r}163 \\
75 \\
103 \\
321 \\
240 \\
53 \\
23 \\
22\end{array}$ \\
\hline $\begin{array}{l}\text { Carbone org. méth. ANNE/mat. organique p. } 1000 \\
\text { Carbone organique (Méthode ANNE) } \\
\text { Matières organiques }\end{array}$ & $\begin{array}{l}19,6 \\
33,7\end{array}$ \\
\hline Azote « KJELDAHL »p. 1000 & 1,22 \\
\hline Rapport carbone organique (ANNE/azote (KJELDAHL) & 16,07 \\
\hline Calcaire total p. 1000 & 0 \\
\hline $\begin{array}{ll}\text { Calcium échangeable } & \text { M. EQ.p. } 100 \\
\text { Cuivre échangeable } & \text { PPM }\end{array}$ & $\begin{array}{l}8,70 \\
0,50\end{array}$ \\
\hline NH4 échangeable $\quad$ M. EQ. p. 100 & 0,44 \\
\hline Cuivre total H.F. & 57 \\
\hline $\begin{array}{l}\text { Carbone et azote des fractions légères } \\
\text { Carbone dans les fractions légères p. } 1000 \\
\text { Carbone des fractions légères dans le sol p. } 1000 \\
\text { Carbone des fractions légères en p. } 1000 \text { du carbone total } \\
\text { Azote p. } 1000 \text { dans les fractions légères } \\
\text { Azote p. } 1000 \text { des fractions légères dans le sol } \\
\text { Azote des fractions légères p. } 100 \text { de l'azote total }\end{array}$ & $\begin{array}{c}165,3 \\
9,02 \\
46,05 \\
8,8 \\
0,48 \\
39,38\end{array}$ \\
\hline Carbone/azote des fractions légères & 18,78 \\
\hline$p H$ & 4,85 \\
\hline
\end{tabular}

L'ensemencement de la biomasse fongique par la microflore tellurique est assuré au départ de l'expérience par l'addition d'eau stérile nécessaire pour ajuster l'humidité du sol expérimental de l'incubateur à 80 p. 100 de sa capacité de rétention. L'eau versée à la surface du cylindre de terre s'enrichit en microorganismes au cours de sa migration et elle atteint la biomasse dans le piège en moins de $2 \mathrm{mn}$.

A des temps d'incubation croissants $(0,2,4,6,8$ et 10 semaines ; 3, 4, 6, 12 et 21 mois) des séries de pièges sont relevées pour recueillir les échantillons de spores en voie de dégradation.

L'étude de la persistance des biomasses sporales est basée sur l'analyse de leur évolution pondérale exprimée en pourcentage de perte de matière sèche et sur l'estimation de leur potentiel d'inoculum par dénombrement des germes viables après mise en culture de suspensions-dilutions d'une fraction déterminée de l'échantillon sur milieu semi-synthétique gélosé (PARKINSON et al., 1971), le potentiel d'inoculum étant exprimé en nombre de propagules vivantes par $\mathrm{g}$ de matière sèche d'inoculum. Pour éviter les contaminations, le milieu semi-synthétique a été supplémenté en chloramphénicol à la concentration de $1 \mathrm{~g} / 1$; dans ces conditions, après 12 mois d'incubation, les dénombre- ments des colonies ont pu être effectués à des dilutions $10^{-9}$ à $10^{-4}$ et la notation de présence ou d'absence à la dilution du $1 / 1000^{\mathrm{e}}$. Pour chacun des pièges nous étudions 4 prélèvements de $0,1 \mathrm{~g}$ de matière humide.

Dans le cas des 2 isolats de $M$. anisopliae nous avons aussi étudié l'évolution du potentiel infectieux des conidiospores sur les larves de Oryctes rhinoceros L. pour le pathotype $\mathrm{N}^{\circ} 51$ et sur les larves de Cetonia aurata $\mathrm{L}$. pour le pathotype $\mathrm{N}^{\circ} 32$ (FARGUES, 1976). Les essais consistent à pulvériser les insectes sous une tour de traitement avec une quantité donnée d'échantillon : $0,1 \mathrm{~g}$ de matière humide, mis en suspension dans $10 \mathrm{ml}$ ou $100 \mathrm{ml}$ d'eau stérile (dilutions respectives de $10^{-2}$ et $10^{-3}$ ). Chaque prélèvement est éprouvé sur un lot de 20 larves, soit 4 lots par piège relevé. L'activité pathogène de ces échantillons a été comparée avec celle de spores fraîchement récoltées éprouvées aux doses respectives de $10^{5}, 10^{6}, 10^{7}$ et $10^{8}$ spores $/ \mathrm{ml}$, toutes conditions égales par ailleurs.

Les données sont soumises à l'analyse de variance, après transformation angulaire lorsqu'il s'agit de proportions (par exemple, taux de mortalité $\mathrm{x}=$ arc

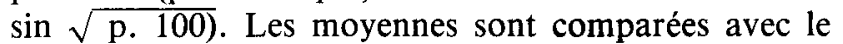
test de Duncan. 


\section{RÉSULTATS}

\section{A. Evolution de la biomasse sporale}

A l'exception de l'isolat $M$. anisopliae $\mathrm{N}^{\circ} 51$ et bien que certains contrôles n'aient pas pu être effectués, on constate que la perte de matière sèche en cours d'incubation des différents inoculums est sensiblement équivalente (tabl. 2).

Après 2 mois d'incubation elle se situe autour de 50 p. 100 (41 p. 100 en 2 mois et 60 p. 100 en 2,5 mois pour les conidies de $B$. bassiana $\mathrm{N}^{\circ} 18$; 55 p. 100 en 2,5 mois pour $M$. anisopliae $\mathrm{N}^{\circ} 32$; 46 p. 100 en 2 mois et 63 p. 100 en 2,5 mois pour $N$. rileyi $\mathrm{N}^{\circ} 5$ et 50 p. 100 en 2 mois pour $P$. fumosoroseus $\mathrm{N}^{\circ} 39$ ) et elle atteint les 90 p. 100 au bout d'un an $\left(87\right.$ p. 100 pour $B$. bassiana $\mathrm{N}^{\circ} 18 ; 96$ p. 100 pour $N$. rileyi $\mathrm{N}^{\circ} 5$ et 98 p. 100 pour $P$. fumoso-roseus $\left.\mathrm{N}^{\circ} 39\right)$. En revanche, la biomasse sporale de $M$. anisopliae $\mathrm{N}^{\circ} 51$ est pratiquement inchangée après 21 mois (tabl. 2).

\section{B. Evolution du potentiel d'inoculurn}

La viabilité des conidies est encore appréciable après 4 ou 6 mois d'incubation daris le sol à $19^{\circ} \mathrm{C}$ (tabl. 2). Néanmoins, la baisse du potentiel d'inoculum des spores de $B$. bassiana $\mathrm{N}^{\circ} 18$ est particulièrement rapide puisque les rapports entre le nombre de germes viables par $\mathrm{g}$ de matière sèche de l'inoculum incubé pendant 4 mois et celui de l'inoculum initial sont de 500 pour $B$. bassiana $N^{\circ} 18$ au lieu de 3 pour les isolats $M$. anisopliae $\mathrm{N}^{\circ} 32, M$. anisopliae $\mathrm{N}^{\circ} 51$ et $N$. rileyi $\mathrm{N}^{\circ} 5$ ou de 0,2 pour $P$. fumoso-roseus $\mathrm{N}^{\circ} 39$ (tabl. 2).
$\mathrm{Au}$ bout d'un an, on note que les potentiels d'inoculum de $B$. bassiana $\mathrm{N}^{\circ} 18$, de $M$. anisopliae $\mathrm{N}^{\circ} 32$, de $N$. rileyi $\mathrm{N}^{\circ} 5$ et de $P$. fumoso-roseus $\mathrm{N}^{\circ} 39$ sont négligeables (inférieurs à $10^{-2}$ propagules vivantes/g de matière sèche) alors que celui de la souche $M$. anisopliae $\mathrm{N}^{\circ} 51$ se maintient au niveau de départ $\left(8.10^{9}\right.$ propagules vivantes $/ \mathrm{g} \mathrm{m}$.s. à $t=0$ et $14.10^{9}$ à $\mathrm{t}=12$ mois). La stabilité du potentiel d'inoculum de l'isolat $\mathrm{N}^{\circ} 51$ est confirmée après presque 2 années d'incubation (11.109 à $\mathrm{t}=21$ mois) (tabl. 2).

\section{Evolution du potentiel infectieux}

Le potentiel infectieux de l'inoculum de $M$. anisopliae $\mathrm{N}^{\circ} 32$ se maintient au cours du $1^{\text {er }}$ mois d'incubation, provoquant 100 p. 100 de mortalité chez les larves contaminées avec une suspension-dilution à $10^{-2}$ $\left(\overline{\mathrm{x}} 30 \mathrm{j}=90 \pm 0\right.$ ) (tabl. 3). Dès le $2^{\mathrm{e}}$ mois, on observe une baisse significative de l'activité pathogène des conidies $(\bar{x} 2$ mois $=62,2 \pm 7,8$, soit une différence par rapport à la mortalité induite par l'inoculum frais de $\Delta=27,8^{* *}$ pour $\Delta 0,01=18,8$ ). Après 3 mois d'incubation la mortalité larvaire n'est plus que d'environ 50 p. $100(\bar{x} 3$ mois $=42,1 \pm 11,3$ ) et, au bout du $4^{e}$ mois d'incubation, elle est inférieure à 5 p. $100(\overline{\mathrm{x}} 4$ mois $=12,1 \pm 14,8)$. Etant donné que la dilution $10^{-2}$ correspond à une suspension titrée à $10^{8}$ spores $/ \mathrm{ml}$, la comparaison de l'activité de l'inoculum en cours d'incubation avec celle de l'inoculum frais (tabl. 3) montre une baisse du potentiel infectieux, exprimé par le rapport des doses létales, de 10 à 100 fois après 3 mois d'incubation $\left(\bar{x} 10^{7}=53,6 \pm 12,5\right.$ soit 65 p. $100>\bar{x} 3$ mois, $10^{-2}=42,1 \pm 11,3$ soit 45 p. $100>\bar{x} 10^{6}=33,0 \pm 5,8$ soit 30 p. 100). Après 4 mois dans le sol expérimental on observe donc à la fois la chute du potentiel infectieux des conidies de l'isolat $\mathrm{N}^{\circ} 32$ (tabl. 3) et celle de leur biomasse (tabl. 2).

\section{TABLEAU 2}

Evolution de la biomasse (perte de matière sèche en p. $100=B$ ) et du potentiel d'inoculum (nombre de colonies par $g$ de matière sèche $=P I$ ) des conidiospores de cultures de surface des pathotypes Beauveria bassiana $N^{\circ} 18$, Metarhizium anisopliae $N^{\circ} 32$ et $N^{\circ} 51$, Nomuraea rileyi $N^{\circ} 5$ et Paecilomyces fumoso-roseus $N^{\circ} 39$ au cours de leur incubation à $19{ }^{\circ} \mathrm{C}$ dans un sol limoneux ajusté à $80 \mathrm{p} .100$ de sa capacité de rétention $(R C=26 \mathrm{p} .100)$.

Variations in dry weight biomass (B) and in inoculum potential (living propagules $/ 1 \mathrm{~g}$ dry fungal material $=$ PI) of aerial conidia of Beauveria bassiana $N^{\circ} 18$, Metarhizium anisopliae $N^{\circ} 32$ et $N^{\circ} 51$, Nomuraea rileyi $N^{\circ} 5$ and Paecilomyces fumoso-roseus $N^{\circ} 39$ during incubation at $19{ }^{\circ} \mathrm{C}$ in a sandy loam, of $26 \%$ water-retention capacity, adjusted to $80 \%$ moisture-holding capacity.

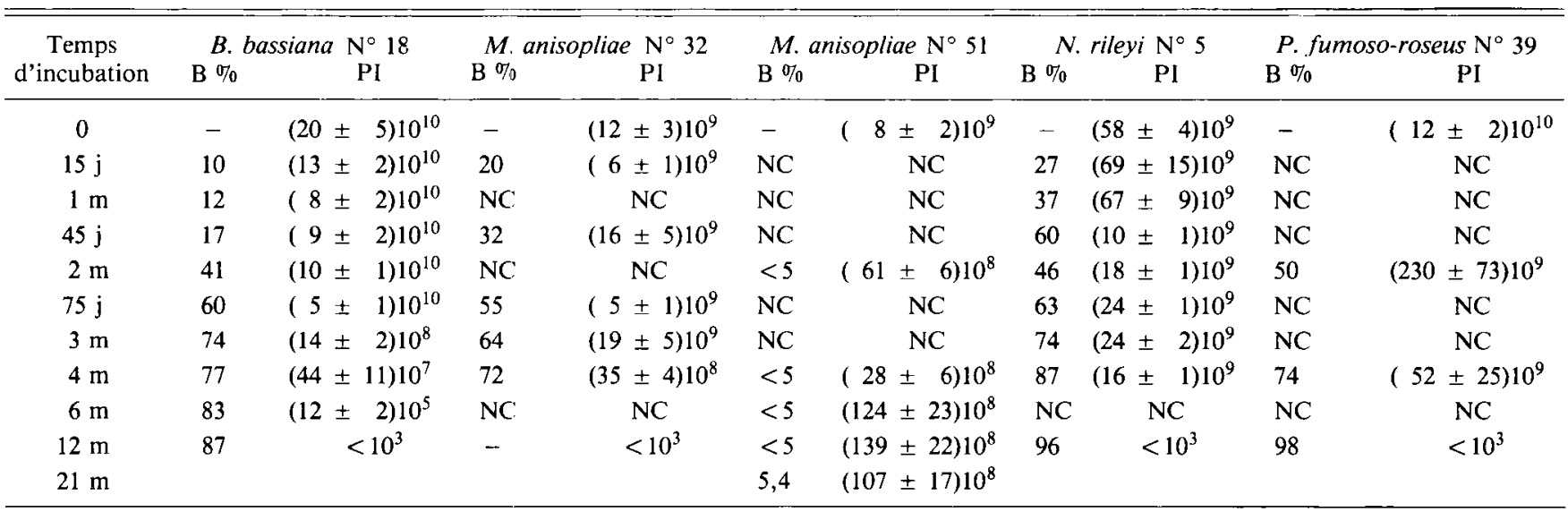




\section{TABLEAU 3}

Evolution du potentiel infectieux, éprouvé sur larves de Cetonia aurata, des conidiospores de Metarhizium anisopliae $N^{\circ} 32$ au cours de leur incubation à $19^{\circ} \mathrm{C}$ dans un sol limoneux ajusté à $80 \mathrm{p} .100$ de sa capacité de rétention.

Infection potential of Metarhizium anisopliae $N^{\circ} 32$ conidia incubated in a sandy loam (adjusted to $80 \%$ moisture-holding capacity) at $19{ }^{\circ} \mathrm{C}$ for $0,1,2,3$ and 4 months, tested on Cetonia aurata larvae (angular values of mortality noted 15 and 30 days after treatment).

\begin{tabular}{cccc}
\hline \hline $\begin{array}{c}\text { Durée d'incubation } \\
\text { dans le sol (mois) }\end{array}$ & $\begin{array}{c}\text { Dilution de l'inoculum } \\
\text { (dose en spores/m) }\end{array}$ & $15 \mathrm{j}$ & Mortalité larvaire (c) \\
\hline 0 (a) & $10^{-2}\left(10^{8}\right)$ & $66,0 \pm 7,6$ & $90 \pm 0$ \\
& $10^{-3}\left(10^{7}\right)$ & $34,6 \pm 6,5$ & $53,6 \pm 12,5$ \\
& $10^{-4}\left(10^{6}\right)$ & $26,5 \pm 2,9$ & $33,0 \pm 5,8$ \\
& $10^{-5}\left(10^{5}\right)$ & $13,5 \pm 9,9$ & $15,9 \pm 11,3$ \\
\hline 1 (b) & $10^{-2}$ & $69,7 \pm 4,9 \mathrm{NS}$ & $90 \pm 0 \mathrm{NS}$ \\
2 (b) & $10^{-2}$ & $55,4 \pm 5,2^{*}$ & $62,2 \pm 7,8^{* *}$ \\
3 (b) & $10^{-2}$ & $28,6 \pm 7,9^{* *}$ & $42,1 \pm 11,3^{* *}$ \\
4 (b) & $10^{-2}$ & $10,3 \pm 12,0^{* *}$ & $12,1 \pm 14,8^{* *}$ \\
\hline
\end{tabular}

(a) Inoculum frais, éprouvé immédiatement après sa récolte à partir des cultures sur milieu semi-synthétique ;

(b) Inoculum en cours d'incubation éprouvé à la dilution $10^{-2}$;

(c) Mortalité cumulée en valeur angulaire ( $\bar{x} \pm$ S.D.) $15 \mathrm{j}$ et $30 \mathrm{j}$ après traitement des insectes; signification de l'effet létal des conidies en cours d'incubation par rapport à celui de l'inoculum frais d'origine (au temps $t=0):\left({ }^{* *}\right)$ hautement significatif ; $\left({ }^{*}\right)$ significatif ; (NS) non significatif.

\section{TABLEAU 4}

Evolution du potentiel infectieux, éprouvé sur larves de Oryctes rhinoceros, des conidiospores de Metarhizium anisopliae $N^{\circ} 5 I$ au cours de leur incubation à $19{ }^{\circ} \mathrm{C}$ dans un sol limoneux ajusté à $80 \mathrm{p} .100$ de sa capacité de rétention.

Infection potential of Metarhizium anisopliae $N^{\circ} 51$ conidia incubated in a sandy loam (adjusted to $80 \%$ moisture-holding capacity) at $19{ }^{\circ} \mathrm{C}$ for $0,2,4,6,12$ and 21 months, tested on Oryctes rhinoceros larvae (angular values of mortality noted 15 and 30 days after treatment).

\begin{tabular}{cccc}
\hline \hline $\begin{array}{c}\text { Durée d'incubation } \\
\text { dans le sol (mois) }\end{array}$ & $\begin{array}{c}\text { Dilution de l'inoculum } \\
\text { (dose en spores/ml) }\end{array}$ & $15 \mathrm{j}$ & $30 \mathrm{j}$ \\
\hline 0 (a) & $10^{-2}\left(10^{8}\right)$ & $86,8 \pm 6,5$ & $90 \pm 0$ \\
& $10^{-3}\left(10^{7}\right)$ & $82,2 \pm 9,3$ & $90 \pm 0$ \\
& $10^{-4}\left(10^{6}\right)$ & $55,4 \pm 14,7$ & $80,8 \pm 10,6$ \\
& $10^{-5}\left(10^{5}\right)$ & $27,5 \pm 16,9$ & $61,1 \pm 11,0$ \\
\hline 2 (b) & $10^{-2}$ & $75,0 \pm 17,3 \mathrm{NS}$ & $90 \pm 0$ \\
4 (b) & $10^{-2}$ & $46,9 \pm 14,0^{* *}$ & $82,5 \pm 0 \mathrm{NS}$ \\
6 (b) & $10^{-2}$ & $90 \pm 0 \mathrm{NS}$ & $90 \pm 0$ \\
12 (b) & $10^{-2}$ & $74,9 \pm 20,0 \mathrm{NS}$ & $90 \pm 0$ \\
21 (b) & $10^{-2}$ & $78,9 \pm 7,8 \mathrm{NS}$ & $90 \pm 0$ \\
\hline 2 (b) & $10^{-3}$ & $15,7 \pm 3,2^{* *}$ & $83,5 \pm 7,5 \mathrm{NS}$ \\
4 (b) & $10^{-3}$ & $21,8 \pm 8,6^{* *}$ & $53,9 \pm 6,4^{* *}$ \\
6 (b) & $10^{-3}$ & $81,1 \pm 11,1 \mathrm{NS}$ & $90 \quad \pm \mathrm{NS}$ \\
12 (b) & $10^{-3}$ & $\mathrm{NC}$ & $\mathrm{NC}$ \\
21 (b) & $10^{-3}$ & $\mathrm{NC}$ & $\mathrm{NC}$ \\
\hline
\end{tabular}

(a) Inoculum frais, éprouvé immédiatement après sa récolte à partir des cultures sur milieu semi-synthétique gélosé ;

(b) Inoculum en cours d'incubation éprouvé aux dilutions $10^{-2}$ et $10^{-3}$;

(c) Mortalité cumulée en valeur angulaire $(\bar{x} \pm$ S.D.) $15 \mathrm{j}$ et $30 \mathrm{j}$ après le traitement des insectes; signification de l'effet létal des conidies en cours de conservation par rapport à celui de-l'inoculum frais d'origine (au temps $t=0$ ) aux dilutions correspondantes. NC $=$ non contrôlé. 
Dans le cas de la souche $M$. anisopliae $\mathrm{N}^{\circ} 51$ (tabl.4), l'activité pathogène de l'inoculum sporal amorce, après 2 mois, une baisse qui est significative à la dilution $10^{-3}$ (mortalité larvaire $15 \mathrm{j}$ après traitement $\overline{\mathrm{x}} 2$ mois $=$ $=15,7 \pm 3,2$ pour $\overline{\mathrm{x}}_{\mathrm{to}}=82,2 \pm 9,3$ bien que $30 \mathrm{j}$ après traitement la différence s'estompe $\overline{\mathrm{x}} 2$ mois $=$ $=83,5 \pm 75$ pour $\bar{x}_{t_{0}}=90 \pm 0$ ). Cette tendance s'affirme après 4 mois d'incubation, puisque les effets létaux des dilutions $10^{-3}$ (équivalent à $10^{7}$ spores $/ \mathrm{ml}$ à $\mathrm{t}=0)$ et $10^{-2}\left(=10^{8}\right.$ spores $/ \mathrm{ml}$ à $\left.\mathrm{t}=0\right)$ ne diffèrent pas significativement des effets de l'inoculum de référence aux doses respectives de $10^{5}$ et $10^{6}$ spores $/ \mathrm{ml}$ (différence entre les taux de mortalité $15 \mathrm{j}$ après traitement $\Delta \mathrm{x}=5,7$ NS pour $\Delta 0,05=15,9$ et $\Delta \mathrm{x}=8,5 \mathrm{NS}$ pour $\Delta 0,05=19,2)$. La perte du potentiel infectieux atteint donc un rapport de 100 au bout de 4 mois, mais la biomasse (matière sèche et potentiel d'inoculum) n'ayant pas évolué significativement depuis le début de l'expérience (tabl. 2) nous avons continué l'analyse de l'activité pathogène de l'inoculum de $M$. anisopliae $\mathrm{N}^{\circ} 51$ maintenu dans le sol. Les contrôles effectués après 6,12 et 21 mois montrent que le potentiel infectieux des spores de l'isolat $\mathrm{N}^{\circ} 51$ retrouve son niveau initial et reste relativement stable (à la dilution $10^{-2}$, mortalités larvaires $15 \mathrm{j}$ après traitement $\overline{\mathrm{x}}_{\mathrm{t}=0}=86,8 \pm 6,3$ soit $99 \mathrm{p} .100$; $\overline{\mathrm{x}} 6$ mois $=90 \pm 0$ soit 100 p. $100 ; \overline{\mathrm{x}} 12$ mois $=74,9 \pm$ 20,0 soit 93 p. 100 et $\bar{x} 21$ mois $=78,9 \pm 7,8$ soit 97 p. $100)$.

\section{DISCUSSION ET CONCLUSION}

Dans les conditions expérimentales utilisées, qui accélèrent les processus de biodégradation des inoculums cryptogamiques entomopathogènes (REISINGER et al., 1977 ; FARGUES et al., 1983), la perte de biomasse sporale est sensiblement identique pour les 4 isolats, $B$. bassiana $\mathrm{N}^{\circ} 18, M$. anisopliae $\mathrm{N}^{\circ} 32, N$. rileyi $\mathrm{N}^{\circ} 5$ et $P$. fumoso-roseus $\mathrm{N}^{\circ} 39$. Toutefois l'analyse de l'évolution des potentiels d'inoculum indique une persistance moindre de $B$. bassiana $\mathrm{N}^{\circ} 18$.

Nos résultats confirment que l'espèce $M$. anisopliae est particulièrement bien adaptée au milieu édaphique (MÜLLER-KÖGLER \& STEIN, 1970, 1976), mais ils démontrent, à travers la comparaison des comportemets respectifs des pathotypes $\mathrm{N}^{\circ} 32(72 \mathrm{p} .100$ de perte de matière sèche en 4 mois) et $N^{\circ} 51$ ( 5 p. 100 de perte en 21 mois), que l'aptitude des conidiospores à survivre dans le sol est inhérente aux propriétés de chaque souche.

L'étude de l'évolution du potentiel infectieux des conidiospores de $M$. anisopliae $\mathrm{N}^{\circ} 51$ au cours de leur conservation a permis de constater une altération de l'activité pathogène des spores après 2 et surtout 4 mois, suivie d'une reprise, avec retour au seuil d'activité initial (tabl. 4). L'activité pathogène étant la propriété la plus fragile de l'inoculum en cours de conservation (FARGUES et al., 1979), il est tout à fait improbable que des spores dont la virulence est altérée retrouvent après plusieurs mois leur activité initiale. Nous pouvons envisager l'hypothèse suivant laquelle les spores seraient capables, au bout d'un certain temps d'incubation dans le sol, de donner de nouvelles propagules dont la virulence serait au moins égale à celle des spores d'origine. L'émission directe d'un conidiophore à partir de spores suivant le processus de germination itérative ayant été décrit chez les hyphomycètes (REISINGER, 1972 ; MANGENOT \& REISINGER, 1976), la poursuite de nos recherches devrait permettre d'étayer cette hypothèse.

En définitive, la persistance d'une souche d'hyphomycète entomopathogène dans le sol ne semble donc pas seulement liée à la résistance de ses conidiospores mais pourrait aussi dépendre de ses facultés à engendrer de nouvelles propagules au cours de microcycles.
Dommergues Y., Mangenot F., 1970. Ecologie microbienne du sol, Masson, Paris, 796 p.

Fargues J., 1976. Spécificité des champignons pathogènes imparfaits (Hyphomycètes) pour les larves de Coléoptères (Scarabaeidae et Chrysomelidae). Entomophaga, 21, 313-233.

Fargues J., Rodriguez-Rueda D., 1980. Sensibilité des larves de Spodoptera littoralis (Noctuidae) aux Hyphomycètes entomopathogènes Nomuraea rileyi et Paecilomyces fumoso-roseus. Entomophaga, 25, 43-54.

Fargues J., Robert P. H., 1983. Effects of passaging through scarabeid hosts on virulence and host specificity of two strains of the entomopathogenic hyphomycete Metarhizium anisopliae. Can. J. Microbiol,.., 29, 576-583

Fargues J., Robert P. H., Reisinger O., 1979. Formulation des productions de masse de l'hyphomycète entomopathogène Beauveria en vue des applications phytosanitaires. Ann. Zool. Ecol. Anim., 11, 247-257.
Fargues J., Cugier J. P., Van de Weghe P., 1980. Expérimentation en parcelles du champignon Beauveria bassiana (Hyphomycète) contre Leptinotarsa decemlineata (Col. Chrysomelidae). Acta Oecol. Oecol. Applic., 1, 49-61.

Fargues J., Reisinger O., Robert P. H., Aubart C., 1983. Biodegra dation of entomopathogenic hyphomycetes : influence of clay coating on Beauveria bassiana blastospore survival in soil. $J$. Invertebr. Pathol., 41, 131-142.

Ferron P., 1971. Problèmes posés par la mise au point d'un procéde de lutte microbiologique contre Melolontha melolontha au moyen de la mycose à Beauveria tenella (Delacr.) Siemaszko. Phytiatr. Phytopharm., 10, 159-168.

Ferron P., 1978. Etiologie et épidémiologie des Muscardines. Thèse Doct. Sci. Nat., Univ. Pierre et Marie Curie, Paris, 114 p.

Ignoffo C. M., Garcia C., Hostetter D. L., Pinnel R. E., 1978. Stability of conidia of an entomopathogenic fungus Nomuraea rileyi in and on soil. J. Invertebr. Pathol., 28, 259-262. 
Joussier D., 1977. Recherches sur Beauveria tenella (Delacr.) Siem. agent de la muscardine des larves du hanneton et son emploi dans la lutte contre cet insecte. Thèse de $3^{\mathrm{e}}$ cycle, Univ., Dijon, $125 \mathrm{p}$.

Kilbertus G., Reisinger O., Mourey A., Cancela da Fonseca J. A., 1975. Biodégradation et humification. Pierron, Sarreguemines, $496 \mathrm{p}$.

Mangenot F., Reisinger O., 1976. Form and function of conidia as related to their development, 789-847. In : Weber D. J., Hess W. M. : The Fungal Spore Form and Function, John Wiley and Sons Inc., 895 p.

Mangenot F., Reisinger O., 1977. Les mécanismes d'altération des champignons phytopathogènes dans le sol, 199-214. In : Travaux dédiés à G. Viennot-Bourgin. Soc. Fr. Phytopathol., $416 \mathrm{p}$.

Müller-Kögler E., Stein W., 1970. Gewächshausversuche mit Beauveria bassiana (Bals.) Vuillemin zur Infektion von Sitona lineatus L. (Coleopt. Curcul.) im Boden. Zeit. Angew. Entomol., 65, 59-76.

Müller-Kögler E., Stein W., 1976. Gewächshausversuche mit Meta- rhizium anisopliae (Metsch.) Sorok. zur Infektion von Sitona lineatus L. im Boden. Zeit. Pflanzenkr. Pflanzensch., 83, 96-108.

Parkinson D., Gray T. A. G., Williams S. T., 1971. Methods for studying the ecology of soil microorganisms. Blackwell Scient. Publ. Oxford and Edinburgh, $116 \mathrm{p}$.

Reisinger O., 1972. Contribution à l'étude ultrastructurale de l'appareil sporifère chez quelques hyphomycètes à paroi mélanisée. Genèse, modification et décomposition. Thèse Doct. Sci. Nat., Univ. Nancy I, 192 p.

Reisinger O., Fargues J., Robert P. H., Arnould M. F., 1977. Effet de l'argile sur la conservation des microorganismes. I. Etude ultrastructurale de la biodégradation dans le sol de l'hyphomycète entomopathogène Beauveria bassiana (Bals.) Vuill. Ann. Microbiol. (Inst. Pasteur), 128, 271-287.

Roberts D. W., Campbell A. S., 1977. Stability of entomopathogenic fungi., M.P.E.A.A.L., 10, 1-80. 